\title{
Orientation Mapping of Extruded Polymeric Composites by Polarized Micro-Raman Spectroscopy
}

\author{
Xiaoyun Chen, ${ }^{1}$ M. Anne Leugers, ${ }^{1}$ Tim Kirch, ${ }^{2}$ and Jamie Stanley ${ }^{1}$ \\ ${ }^{1}$ Analytical Sciences, Core R\&D, The Dow Chemical Company, Midland, MI 48667, USA \\ ${ }^{2}$ Dow Building Solutions, The Dow Chemical Company, 200 Larkin 1605 Joseph Drive, Midland, MI 48674, USA \\ Correspondence should be addressed to Xiaoyun Chen; xchen4@dow.com
}

Received 5 August 2014; Accepted 8 September 2014

Academic Editor: Jie-Fang Zhu

Copyright (C) 2015 Xiaoyun Chen et al. This is an open access article distributed under the Creative Commons Attribution License, which permits unrestricted use, distribution, and reproduction in any medium, provided the original work is properly cited.

\begin{abstract}
Molecular orientation has a strong influence on polymeric composite materials' mechanical properties. In this paper we describe the use of polarized micro-Raman spectroscopy as a powerful tool to map out the molecular orientation of a uniaxially oriented polypropylene- (PP-) based composite material. Initial samples exhibited a high degree of surface fibrillation upon cutting. Raman spectroscopy was used to characterize the degree of orientation in the skin and guide the development of the posttreatment process to optimize the skin relaxation while maintaining the high degree of orientation in the rest of the board. The PP oriented polymer composite (OPC) was oriented through an extrusion process and its surface was then treated to achieve relaxation. Micro-Raman analysis at the surface region demonstrated the surface orientation relaxation, and the results provide an effective way to correlate the extent of relaxation and process conditions. Larger scale orientation mapping was also carried out over the entire cross-section $(12.7 \mathrm{~cm} \times 2.54 \mathrm{~cm})$. The results agree well with prior expectation of the molecular orientation based on the extrusion and subsequent quenching process. The methodologies described here can be readily applied to other polymeric systems.
\end{abstract}

\section{Introduction}

Polymer composites are gaining market share in construction markets and offer several advantages over the traditional wood materials. A variety of plastic composite manufacturers are offering products for the decking, window and door, and other specialty markets. A recent development is an oriented polymer composite (OPC). Using a temperature controlled proprietary orientation process, products can be fabricated with a unique combination of low density, high stiffness, good processability with traditional wood-working tools, and weatherability compared to traditional plastic composite structures. Unlike wood boards, where the orientation of the fibrils and microfibrils is aligned by nature to maintain stiffness, semicrystalline polymeric materials must be oriented during processing to align the polymer chains to achieve the desired stiffness.

A high degree of orientation of amorphous regions and crystalline regions is quite desirable for modulus and stiffness under load; however the orientation can present issues with the shaping and cutting of the composites to form the final part. Initial products showed a high degree of orientation on the surface of the product that extended toward the center of the parts dimensions. These products exhibited highly undesirable fibrillation during surface wear evaluation and cutting. Efforts were initiated to understand and improve both wear and cutting performance.

Extensive studies have been previously carried out to characterize molecular orientation of a wide range of systems such as liquid crystals, polymers, proteins, and peptides using vibrational spectroscopic tools. For example, polarized infrared spectroscopy (IR) can be used to obtain order parameter defined as $S_{\theta}=\left(3\left\langle\cos ^{2} \theta\right\rangle-1\right) / 2$, where $\theta$ is the angle of the functional group's dipole moment relative to the symmetry axis and the bracket denotes ensemble average [1]. However, the requirement of thin samples sections for transmission mode or a good contact with attenuated total reflectance (ATR) crystals in the ATR mode limits the application of IR for molecular orientation study in an industrial environment. Polarized Raman spectroscopy offers more flexibility as signals can be generated from any surface or cross-section in a contact-free fashion and, in theory, yields 
more information. For example, the second and fourth terms of the orientation distribution function, characterized by Legendre polynomial $\left\langle P_{2}(\cos \theta)\right\rangle$ and $\left\langle P_{4}(\cos \theta)\right\rangle$, can be calculated for vibrational modes whose Raman tensor is known $[2,3]$. Other higher order spectroscopy techniques such as second harmonic generation (SHG) $[4,5]$ and sum frequency generation vibrational spectroscopy (SFG) [6-8] have also been shown to provide insights into molecular orientation, especially when combined with linear spectroscopy techniques such as IR or Raman. Their complex instrumentation and data analysis and surface/interface-specific nature have limited their applications mostly to academic settings.

Here we describe an industrial application of polarized Raman spectroscopy to characterize the molecular orientation of polymers within an oriented composite material with uniaxial orientation. It is demonstrated that through the use of orientation ratio (O.R. (1)), the molecular orientation of OPC can be effectively characterized on length scale spanning from $\mu \mathrm{m}$ scale to macroscopic scale, thus enabling the mapping of orientation across the entire cross-section of an OPC sample. O.R. is defined in (1), where $I_{1}$ stands for the intensity of a band that is sensitive to polarization while $I_{2}$ stands for the intensity of another band that is insensitive to polarization or shows the opposite trend. The $Z Z$ and $Y Y$ in the subscript denote whether the spectrum is collected with the electric field of the excitation/collection beam parallel or perpendicular to the extrusion direction of the sample (see Figure 1):

$$
\text { O.R. }=\frac{I_{1, Z Z} / I_{2, Z Z}}{I_{1, Y Y} / I_{2, Y Y}} .
$$

Although this approach does not yield detailed quantitative orientation distribution functions, as the more elaborate polarized Raman methods previously developed, its simplicity and robustness make it suitable for industrial applications where most samples have many nonpolymer components such as additives, fillers, and pigments. Voyiatzis and Andrikopoulos showed that despite the use of calibration curve, the O.R. can potentially be correlated to the various terms of the orientation distribution function [9]. This was not necessary for the applications investigated herein. Voyiatzis and Andrikopoulos also demonstrated that, for well-behaved systems such as a dye dissolved in polymeric matrix, the orientation of the dye molecules can be effectively characterized by a simple ratio of $I_{1, z z} / I_{2, z z}$, which enables fast monitoring of orientation during a drawing process. The assumptions behind this approach may not always be satisfied by many industrial OPC samples. For example, difference in crystallinity may be another factor that may cause a change in the $I_{1, z z} / I_{2, z z}$ ratio $[10,11]$. The $I_{1, y y} / I_{2, y y}$ term in the denominator of the O.R. equation can effectively account for the variation of other factors such as crystallinity and concentration.

\section{Materials and Methods}

2.1. OPC Board Production. The details on the OPC extrusion and processing can be found in previous patents [12].
An appropriate formulation, consisting of both miscible and nonmiscible components, is extruded into a profile using standard material feeding and extruding technology. The extruded profile is then uniaxially drawn using a drawing die in a temperature controlled environment. The ratio of the product dimensions before and after drawing is known as the drawing ratio. As the drawing ratio is increased towards its optimum value, density tends to decrease while product stiffness, defined by flexural modulus and elasticity, tends to increase.

Use of high draw ratios tends to produce products with high degrees of surface orientation. While the level of orientation generates many useful properties for the oriented polymer composite, high surface orientation causes issues when the product's surface undergoes surface wear or the product is cut to specific lengths or shapes. As noted earlier, the high degree of orientation tends to generate fibrils on the worn surface or along the fabricated edges of the product. Experimentation indicated that controlled surface deorientation would eliminate these issues. An analytical technique was required that could determine the level of orientation in multiple axis to allow identification of an effective heattreating method to achieve surface deorientation as well as develop control parameters for processing equipment that could be applied during the commercial manufacturing process.

2.2. Sample Preparation for Micro-Raman Spectroscopy. An OPC board was cut with a saw along the extrusion direction to expose the cross-section that was perpendicular to the heat-treated surface as shown in Figure 1. The cutting direction shown in Figure 1 is vertical but can also be horizontal as long as it is parallel to the extrusion direction. The exposed cross-section was then ground to a smooth flat surface using grinding papers of $320,800,1200,2400$, and 4000 grit on a Struers Pedemat Rotopol-V polisher with water cooling. Control experiments using different orientation of samples relative to polishing direction were carried out to ensure that such sample preparation method does not alter or introduce orientation to OPC board cross-sections. Polarized Raman spectra were then collected at various depths as indicated by the red dots on the polished cross-section as shown in Figure 1.

\subsection{Polarized Micro-Raman Analysis. A Kaiser Raman RXN1} microscope with $785 \mathrm{~nm}$ laser excitation was used. A schematic of the Raman optical pathway is shown in Figure 1. Multimode optical fibers were used to deliver the laser to the microscope and the signal back to the spectrometer. Two independent linear polarizers were used to control the polarization state of the excitation laser and the Raman signals.

Raman signals were collected in the backscattering geometry using a 20x long-working-distance objective. The laser spot size was approximately $20 \mu \mathrm{m}$ in diameter. Typical laser power used was in the range of 30 to $100 \mathrm{~mW}$, depending on the pigment loading in a sample. It should be noted that laser heating could cause molecular relaxation and even melting. The exact power used for each spectrum was generally half of 


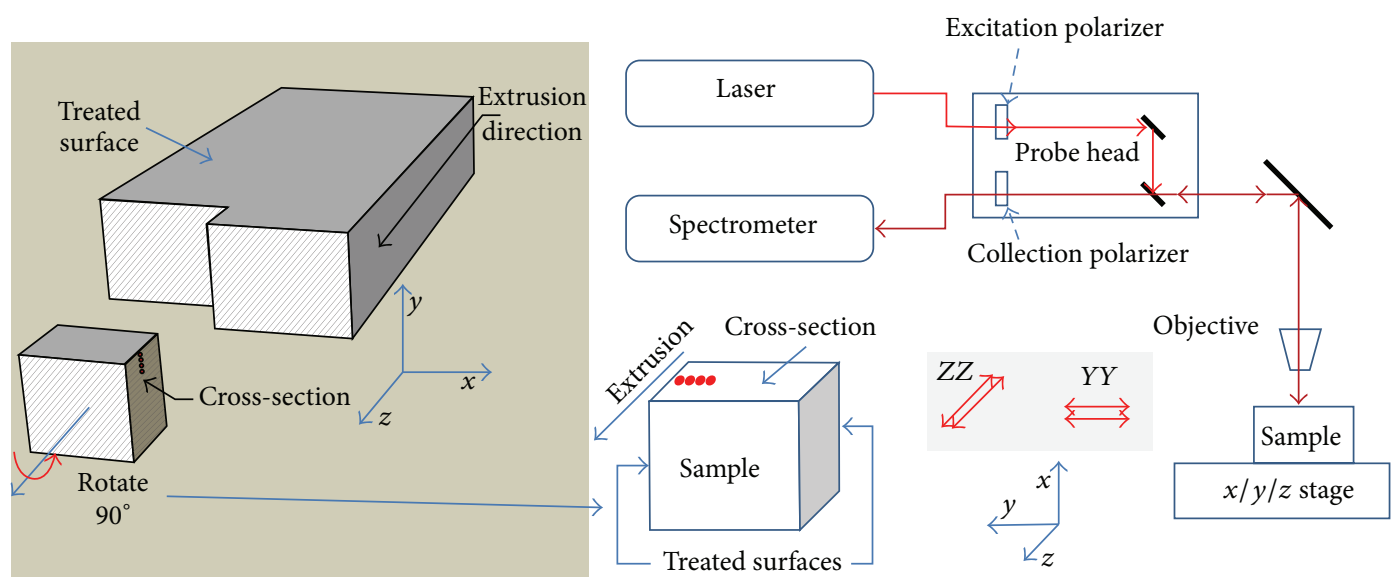

FIGURE 1: Schematic illustration of how a board was cut and analyzed by micro-Raman analysis.

the power that would cause specimen surface melting. One minute collection time was used to acquire sufficient signalto-noise ratio.

At each point of a sample two spectra were collected, the parallel and the perpendicular. The parallel (perpendicular) spectrum is collected with the two polarizers oriented so that the electric field of the incoming laser beam and the outgoing signal beam are both parallel (perpendicular) to the extrusion direction. It should be noted that that there are many more geometries that can be used (e.g., one polarizer parallel to and one polarizer perpendicular to the extrusion direction, or with the sample oriented differently), but the parallel and perpendicular spectra collected in the manner described above can best reflect the orientation differences in a sample.

\section{Results and Discussion}

3.1. Control Samples. Figure 3 compares the parallel and perpendicular spectra collected from a plastic pellet of the same material used in the composite formulation (top panel) and from an oriented fiber of the same material (bottom panel). For this specific sample, polypropylene was used as the plastic component of the composite. Details in the peak assignments for PP have previously been reported and will not be repeated here $[13,14]$. It is obvious that while the parallel and perpendicular spectra are almost indistinguishable for the PP pellet sample (note all spectra are normalized to the strongest peak within each panel), dramatic differences can be found for the parallel and perpendicular spectra of PP fibers.

Such differences in the spectra collected with different polarizers are commonly observed for most oriented polymers and several examples are shown in Figure 3. The extent of differences exhibited by the parallel and perpendicular spectra depends on several factors, such as the level of orientation within the macromolecular chain, the orientation of a functional group relative to its polymer backbone, and the Raman tensor of the functional group. As can be observed in Figure 3, spectral differences present between the parallel and perpendicular spectra range from dramatic for polyethylene and poly(vinylidene fluoride-co-polyester) fibers, to intermediate for polybutene-1 fibers, and to quite subtle for poly(styrene-co-acrylonitrile) fibers. O.R. should not be used to directly compare the level of orientation across different polymers and rather should only be used as a relative measure to evaluate molecular orientation level for uniaxially oriented samples of the same polymer. Discussion below will be focused on the PP orientation in OPC boards.

According to (1), two bands need to be selected for the calculation of O.R. for each phase. For the PP investigated here, both the crystalline and the amorphous phases are present in significant amount and therefore two sets of bands should be used, one set for each phase [15]. Below we will focus on the orientation characterization of the crystalline phase because a similar trend was observed for the amorphous phase. The baseline-corrected peak height of the $809 \mathrm{~cm}^{-1}$ peak and the $841 \mathrm{~cm}^{-1}$ peak is used as $I_{1}$ and $I_{2}$, respectively, as used in several previous studies [16-18]. In the next sections line maps, small-area mapping and largearea mapping will be shown for the PP OPC samples with and without surface treatment.

3.2. Line Maps. For consistency, PP OPC cross-section samples are always oriented the same way in reference to the lab coordinate system with the extrusion direction parallel to the microscope stage as shown in Figure 1. Two line maps were collected: one parallel and one perpendicular. A stable stage is critical to ensure that the corresponding parallel and perpendicular spectra in the two line maps are from the same spot. Spectra in these line maps were collected from evenly spaced points on an OPC cross-section spanning from the surface into the core. The O.R. profiles from three representative samples are shown in Figure 4. Samples (a), (b), and (c) were from similar OPC boards, but after different level of surface treatments aimed to cause orientation relaxation, with (a) receiving no surface treatment, (b) an intermediate level, and (c) the highest level of surface treatment. Before we compare the O.R. profiles in Figure 4, it is worth noting that the O.R. for the PP fiber shown in Figure 2 is calculated to be 7.7 


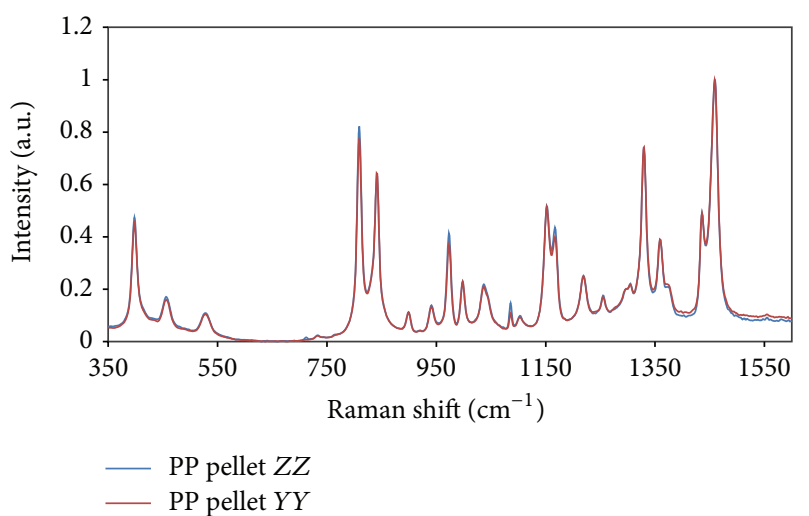

(a)

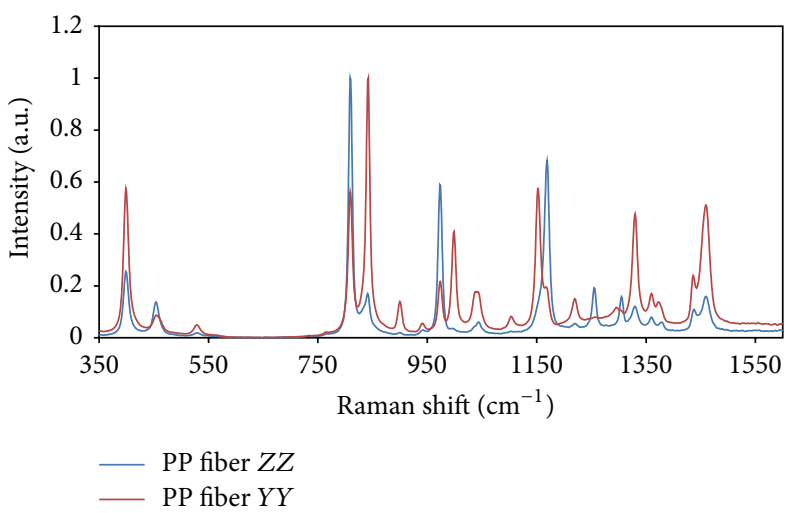

(b)

Figure 2: (a) $Z Z$ and $Y Y$ spectra of a PP pellet; (b) $Z Z$ and $Y Y$ spectra of a PP fiber.

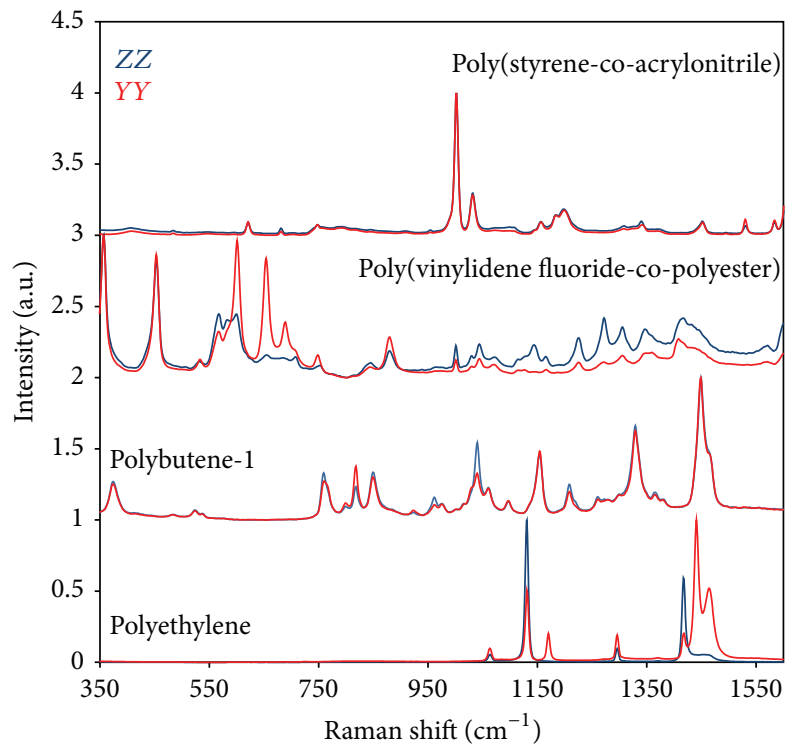

Figure 3: Comparison of $Z Z$ to $Y Y$ spectra of (from top to bottom) poly(styrene-co-acrylonitrile), poly(vinylidene fluorideco-polyester), poly(1-butene), and polyethyelene.

and about 1 for the PP pellet. The values of 7.7 and 1 are specific for the particular bands selected $\left(841\right.$ and $\left.809 \mathrm{~cm}^{-1}\right)$ and other choices of bands may also be valid but will have different O.R. values. The O.R. ratio calculated using the same pair of bands for PP in OPC can be compared. It is unlikely for the O.R. value for PP in an extrusion oriented sample to reach as high as 7.7 as very high draw ratio is needed; for completely relaxed PP samples its O.R. will be close to 1 as the polarization should not affect the Raman spectra of isotropic materials. If somehow the PP chain has a preferred orientation in the transverse direction, then an O.R. smaller than 1 will be observed. Samples with O.R. value of $x$ and $1 / x$ should have the same level of orientation, but with the orientation direction orthogonal to each other.

Given the above information, it is clear from Figure 4 that while the inner region of all three samples is relatively orientated with O.R. ranging from 2.5 to 4 , their surface orientation exhibited dramatic differences. Sample (a) showed no relaxed orientation at the surface because it did not receive any surface treatment. Samples (b) and (c) both showed partially relaxed surface region with O.R. ranging between 1 and 1.6, but the relaxed surface layer is only about $110 \mu \mathrm{m}$ for Sample (b), much thinner than the $380 \mu \mathrm{m}$ thick relaxed skin of Sample (c). Such observations are in good agreement with the sample treatment history and provided an effective way to understand how efficient the treatment is in relaxing polymer orientation.

3.3. Small-Area Maps. The above line map methods can be readily applied to acquire an area map of PP orientation if the area of interest can fit within the range of the microscope stage. It is critical for the OPC cross-section surface to be horizontal and parallel to the stage to ensure that all the spots in the map are in focus. The results shown in Figure 5 were from a surface-treated OPC cross-section with its surface on the right edge of the map. Spectra were collected in a raster-scan fashion from an area of $600 \mu \mathrm{m}$ (along the depth direction) $\times 1000 \mu \mathrm{m}$ (along the extrusion direction). The red dots in Figure 5 right panel represent the spectral collection spots, which formed a $26 \times 8$ grid, with 26 evenly spaced lines distributed along the depth direction and 8 evenly spaced lines distributed along the extrusion direction. Such a form of grid was chosen in order to capture the sharp transition between the skin and core region and because less dramatic change in O.R. was expected along the extrusion direction. The map in Figure 5 indeed confirms this expectation and clearly shows that the skin layer was relatively uniform with a thickness on the order of 200 to $250 \mu \mathrm{m}$. The false-color map in the left panel of Figure 5 was generated by interpolating the O.R. over the $26 \times 8$ grid.

3.4. Large-Area Mapping. While the results above clearly demonstrate the power of polarized micro-Raman analysis to characterize the surface relaxation, the orientation distribution across the entire OPC product cross-section is what determines the overall mechanical strength of an OPC 


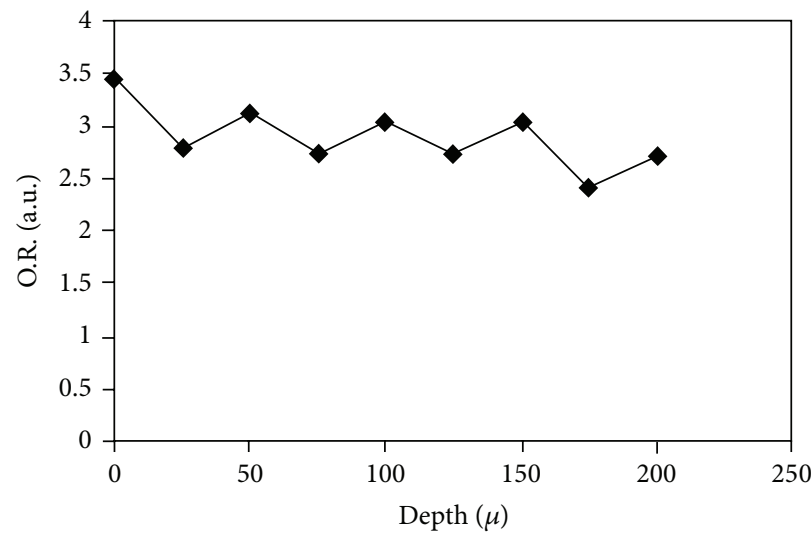

(a)

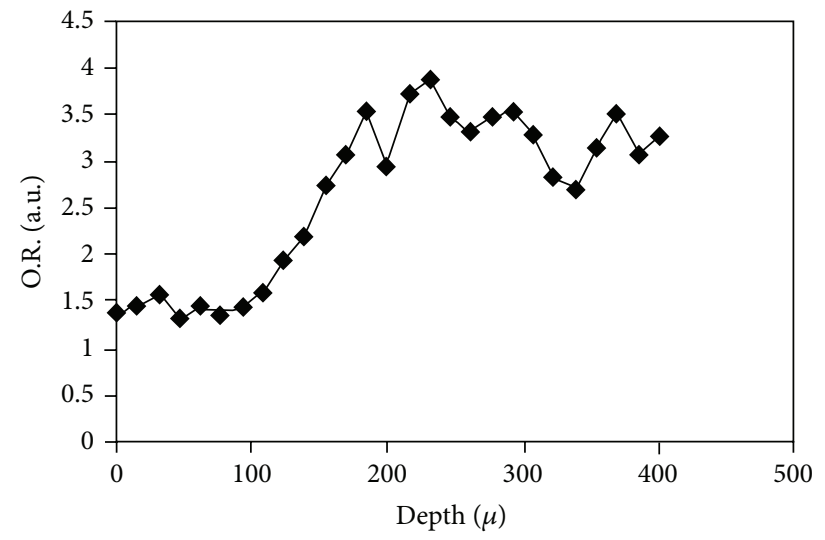

(b)

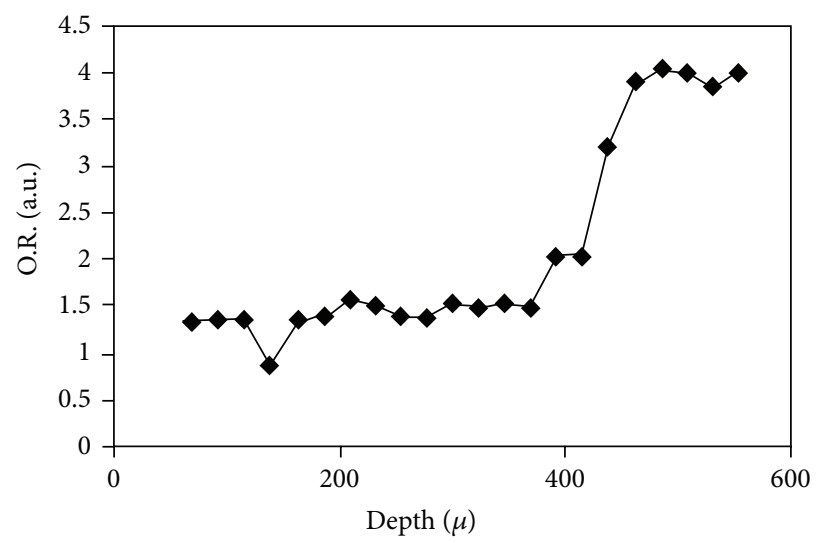

(c)

FIGURE 4: Line maps of O.R. profiles collected from the surface region of three different samples with (a) no, (b) medium level, and (c) high level of surface treatment.
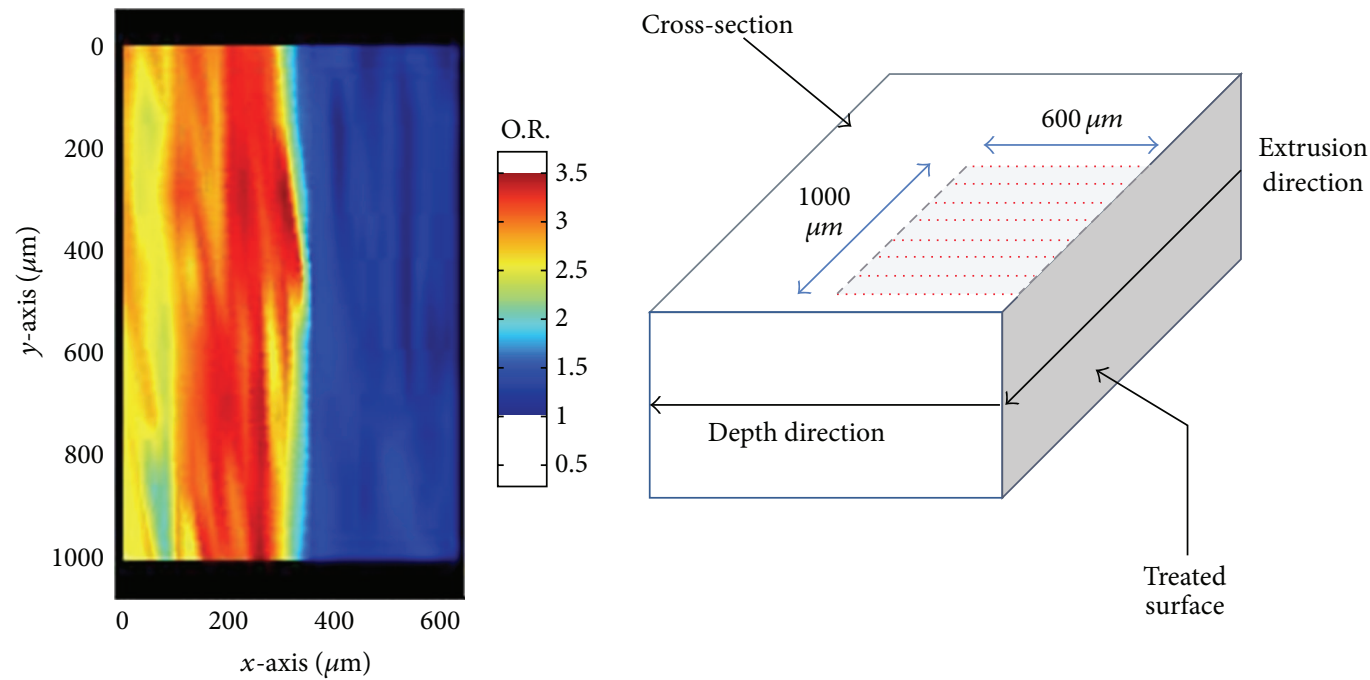

FIGURE 5: Mapping of orientation for a $600 \times 1000 \mu \mathrm{m}$ area as marked by the shaded area with red dots representing the spectral collection spots. 
structure. Many interdependent factors can influence the final strength of an OPC product such as the die design, temperature, feed rate, cooling rate, and fillers and it is often difficult to understand how these various factors can lead to final product performance without first understanding how they influence the molecular orientation.

Figure 6 illustrates how the large-area orientation mapping was carried out. An OPC sample was cut into 11 vertical sections and line maps were carried out on each section as represented by the red dots. The sample can also be cut into horizontal sections. However, due to the sample geometry, horizontal sections are thin and thus more prone to deformations.

The orientation mapping results from two representative samples are shown in the bottom panel of Figure 6. OPC sample 1 was a regular product while a side slot was built into OPC sample 2 according to its design. The orientation mapping yields a direct and visual way to understand the level of orientation at various regions within an OPC product. Both samples showed similar general patterns: a surface skin region on the top and bottom of the product that underwent relaxation during surface treatment, a relatively oriented region beyond the skin, and a relaxed core region.

For OPC sample 1 the entire orientation map shows remarkable symmetry along both the horizontal and vertical middle line, with the oriented outer layer about $2.5 \mathrm{~cm}$ or 1 inch thick (excluding the relaxed surface which was oriented prior to surface treatment) surrounding the core region and with the core region showing a gradient of O.R. which can be further used to define an inner and outer core region. Such a distribution of orientation supports the hypothesis that the core region, due to insulation by the outer layer, has more time to relax than the material in the outer layer. In contrast the outer region cools down faster and thus effectively locks the molecular orientation once the sample temperature drops below $T_{m}$ or $T_{g}$. Analysis using micro-Raman technology provides an improved understanding of how process conditions can be related to molecular orientation and ultimately to product performance which is thus achieved. OPC sample 2 shows an orientation map that is rather different from OPC sample 1. This sample appeared to be less oriented overall. The exact reasons behind such differences are of great interest to engineers and material scientists but are beyond the scope of this study.

\section{Conclusions}

In this study we used polarized micro-Raman spectroscopy to study PP morphology and orientation in uniaxially oriented OPC products in order to guide the posttreatment of PP composite products. We applied this method to analyze PP $\mathrm{OPC}$ and the results from the surface region enable us to correlate the surface treatment parameters to the level of skin orientation relaxation and skin thickness, and the results from the large scale mapping show in a visual way how process conditions can influence the molecular orientation across the entire cross-section of an OPC material.

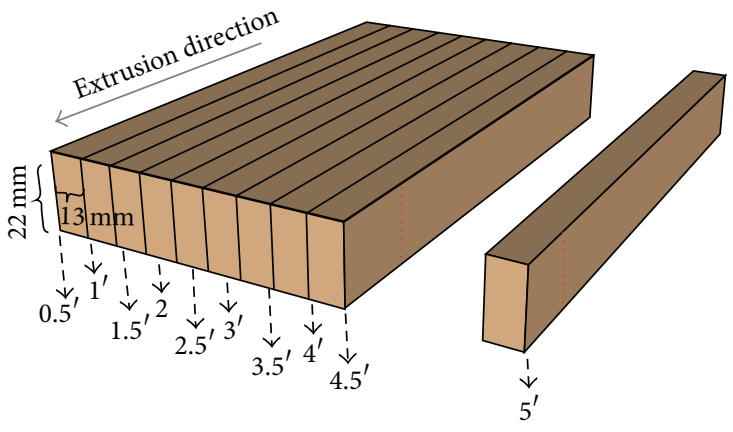

(a)
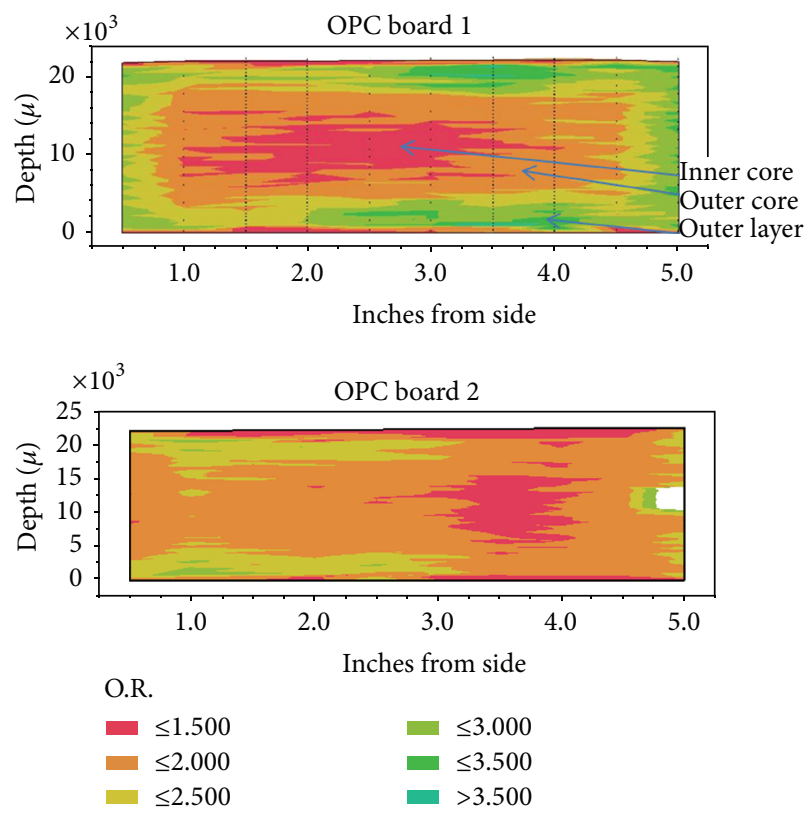

(b)

FIgURE 6: (a) A schematic illustration of how an OPC board is cut into sections. (b) Orientation map of two OPC boards. O.R. is colorcoded.

The methodologies described here can be readily applied to other polymeric systems.

\section{Conflict of Interests}

The authors declare that there is no conflict of interests regarding the publication of this paper.

\section{Acknowledgments}

The authors would like to thank the Dow Chemical Company and Eovations, LLC, for permission to publish this work. Help in sample preparation from Robbie Samson is gratefully acknowledged. They also appreciate the inspiring discussions with Robert Cieslinski and Kevin Nichols. 


\section{References}

[1] L. K. Tamm and S. A. Tatulian, "Infrared spectroscopy of proteins and peptides in lipid bilayers," Quarterly Reviews of Biophysics, vol. 30, no. 4, pp. 365-429, 1997.

[2] M. Tanaka and R. J. Young, "Review Polarised Raman spectroscopy for the study of molecular orientation distributions in polymers," Journal of Materials Science, vol. 41, no. 3, pp. 963991, 2006.

[3] R. Ikeda, B. Chase, and N. J. Everall, "Basics of orientation measurements in Infrared and Raman spectroscopy," in Handbook of Vibrational Spectroscopy, John Wiley \& Sons, 2006.

[4] T. G. Zhang, C. H. Zhang, and G. K. Wong, "Determination of molecular orientation in molecular monolayers by secondharmonic generation," Journal of the Optical Society of America B, vol. 7, no. 6, pp. 902-907, 1990.

[5] M. B. Feller, W. Chen, and Y. R. Shen, "Investigation of surface-induced alignment of liquid-crystal molecules by optical second-harmonic generation," Physical Review A, vol. 43, no. 12, pp. 6778-6792, 1991.

[6] X. Chen, M. L. Clarke, J. Wang, and Z. Chen, "Sum frequency generation vibrational spectroscopy studies on molecular conformation and orientation of biological molecules at interfaces," International Journal of Modern Physics B, vol. 19, no. 4, pp. 691713, 2005.

[7] Z. Chen, Y. R. Shen, and G. A. Somorjai, "Studies of polymer surfaces by sum frequency generation vibrational spectroscopy," Annual Review of Physical Chemistry, vol. 53, pp. 437-465, 2002.

[8] X. Chen, J. Wang, A. P. Boughton, C. B. Kristalyn, and Z. Chen, "Multiple orientation of melittin inside a single lipid bilayer determined by combined vibrational spectroscopic studies," Journal of the American Chemical Society, vol. 129, no. 5, pp. 1420-1427, 2007.

[9] G. A. Voyiatzis and K. S. Andrikopoulos, "Fast monitoring of the molecular orientation in drawn polymers using microRaman spectroscopy," Applied Spectroscopy, vol. 56, no. 4, pp. 528-535, 2002.

[10] C. Minogianni, K. G. Gatos, and C. Galiotis, "Estimation of crystallinity in isotropic isotactic polypropylene with Raman spectroscopy," Applied Spectroscopy, vol. 59, no. 9, pp. 1141-1147, 2005.

[11] R. M. Khafagy, "In situ FT-Raman spectroscopic study of the conformational changes occurring in isotactic polypropylene during its melting and crystallization processes," Journal of Polymer Science, Part B: Polymer Physics, vol. 44, no. 15, pp. 2173-2182, 2006.

[12] J. J. O’Brien, T. O. Kirch, K. L. Nichols, and B. M. Birchmeier, "Oriented polymer composition with a deoriented surface layer," US8475700 B2, 2010.

[13] M. C. Tobin, "The infrared spectra of polymers. III. The infrared and Raman spectra of isotactic polypropylene," The Journal of Physical Chemistry, vol. 64, no. 2, pp. 216-219, 1960.

[14] P. D. Vasko and J. L. Koenig, "Raman scattering and polarization measurements of isotactic polypropylene," Macromolecules, vol. 3, no. 5, pp. 597-601, 1970.

[15] R. P. Paradkar, A. Leugers, R. Patel, X. Chen, G. Meyers, and E. Lipp, "Raman spectroscopy of polymers," in Encyclopedia of Analytical Chemistry, John Wiley \& Sons, New York, NY, USA, 2006.

[16] D. García-López, J. C. Merino, I. Gobernado-Mitre, and J. M. Pastor, "Polarized confocal Raman microspectroscopy studies of chain orientation on injected poly(propylene)/montmorillonite nanocomposites," Journal of Applied Polymer Science, vol. 96, no. 6, pp. 2377-2382, 2005.

[17] X. Wang and S. Michielsen, "Isotactic polypropylene morphology-Raman spectra correlations," Journal of Applied Polymer Science, vol. 82, no. 6, pp. 1330-1338, 2001.

[18] K. Aratake, M. Taniguchi, and H. Ito, "Polyolefin fiber and nonwoven fabric produced by using the same," Patent: US5910362 A, 1999. 

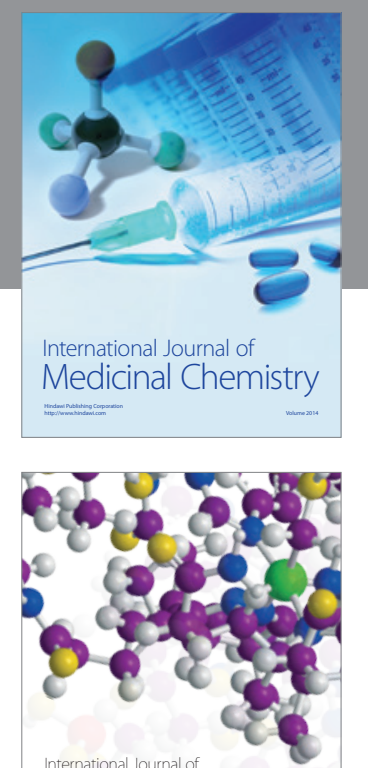

\section{Carbohydrate} Chemistry

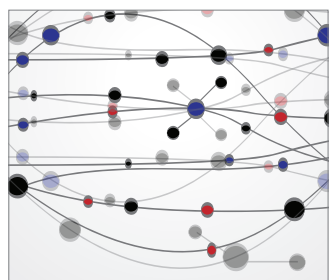

The Scientific World Journal
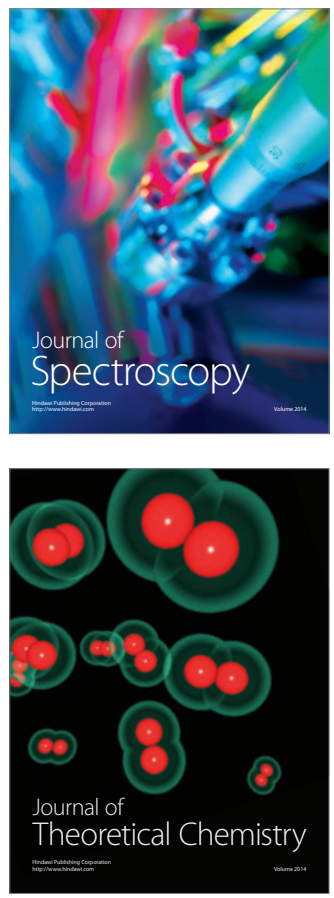
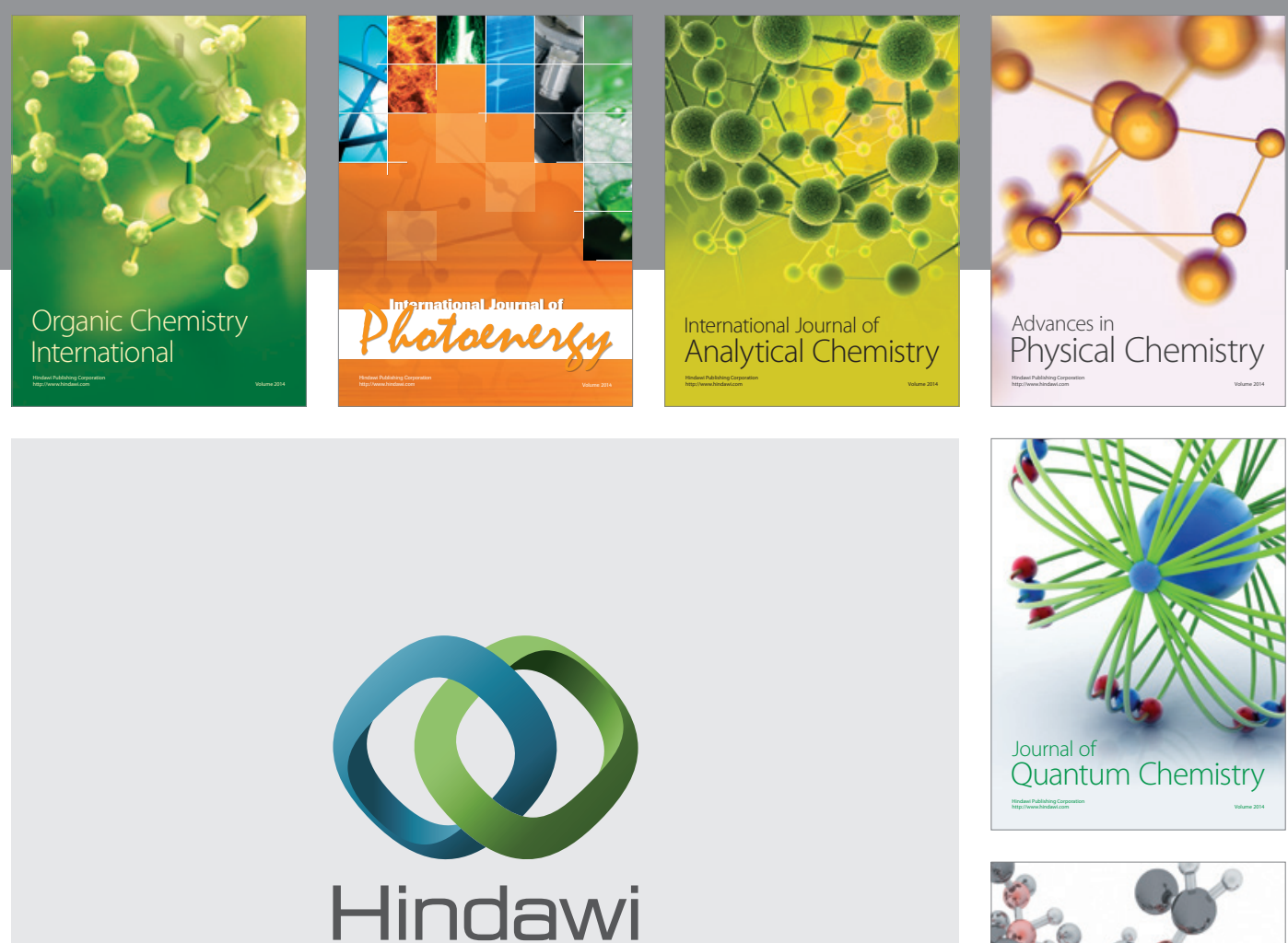

Submit your manuscripts at

http://www.hindawi.com

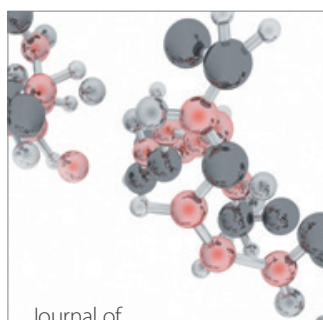

Analytical Methods

in Chemistry

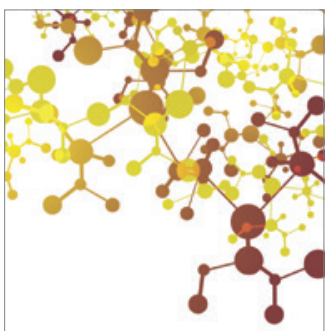

Journal of

Applied Chemistry

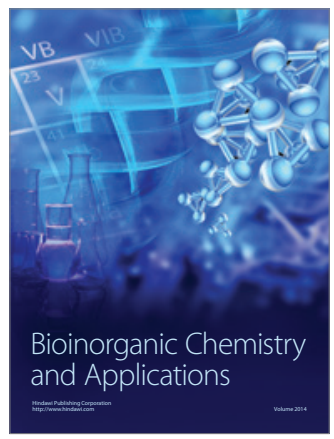

Inorganic Chemistry
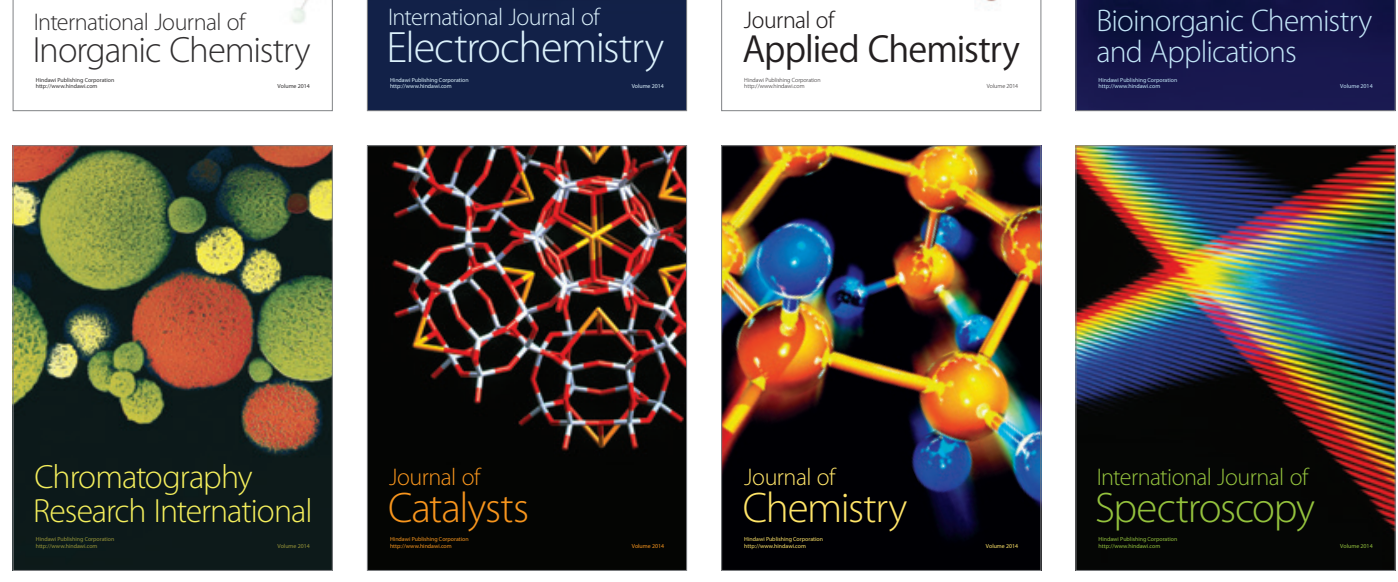